\title{
Enhanced Dynamic Authorized Secured Protocol for Wireless Sensor Networks
}

\author{
Dr.J.R.Arunkumar
}

Faculty of Computing and Software Engineering,AMIT,Arbaminch University,Ethiopia.

\begin{tabular}{lll}
\hline \multicolumn{3}{l}{ Article Information } \\
Received $\quad:$ & 14 March 2020 \\
Revised & $:$ & 19 March 2020 \\
Accepted & $:$ & 24 March 2020 \\
Published & 10 April 2020
\end{tabular}

Corresponding Author:

Dr.J.R.Arunkumar

Email:arunku@yahoo.com

\begin{abstract}
In Wireless Sensor Networks arrange is the technique for transferring new code or modifying the reasonableness of existing code. For security reasons, each code update ought to be authenticated to ignore unauthenticated from mounting malignant code with in the Wireless Sensor Networks. Totally existing reinventing conventions depend on the incorporated technique wherein single the base station has the position to inductee rewrite the programming. On the other hand, it is required and some of the time required for different approved system clients to simultaneously and straightforwardly reinvent sensor nodes while excluding the base station, which is referenced to as disseminated reconstructing of program. The system vender can even allot distinctive reinventing benefits to various clients Very as of late, a novel ensured and appropriated reconstructing program convention named Enhanced Secure Dynamic Authorized Secured protocol has been proposed, which is the principal exertion of its sort. Alternately, right now, distinguish a trademark configuration deficiency in the client preprocessing period of EDASP and approve that it is defenseless to a pantomime assault by which an enemy can basically imitate any approved client to finish reinventing. Thusly, we propose a straightforward adjustment to fix the perceived security issue without losing any highlights of. The Node c Enhanced Dynamic Authorized Secured protocol order calculation is utilized to sort the sensor hub before transmitting the code picture. Every single client need to confirm the sensor in its benefit list before sending the code picture.
\end{abstract}

Keywords: EDASP, Node protocol, security, wireless sensor networks.

Copyright @ (2020: Dr.J.R.Arunkumar. This is an open access distribution, and reproduction in any medium, provided Access article distributed under the Creative Commons Attribution License the original work is properly cited License, which permits unrestricted use.

Citation: Dr.J.R.Arunkumar. "Enhanced Dynamic Authorized Secured Protocol for Wireless Sensor Networks, "Journal of Science, Computing and Engineering Research, 1(1), 07-11, Mar-Apr 2020.

\section{INTRODUCTION}

A wireless sensor network (WSN) includes of spatially conveyed free sensors to show physical or natural conditions. The more present day systems are bi-directional, likewise permitting control of sensor movement. The improvement of WSNs was empowered by military applications, for example, front line examination. The remote sensor arrange is worked of "Nodes" - from a couple to a large number or even thousands, where every hub is associated. : Wireless reconstructing is that the technique for spreading another code picture or significant directions to detecting component hubs through remote connections when a remote detecting component arrange (WSN) is sent. To the necessity of evacuating bugs and including new functionalities, reconstructing is a significant activity perform of WSN. As a WSN is ordinarily sent in antagonistic conditions like the field of fight, a foe may abuse the reinventing component to dispatch different assaults. In this way, secure writing computer programs is and can keep on being a huge concern.

There has been a lot of examination that spend significant time in secure reconstructing, and numerous eye catching conventions are anticipated lately Notwithstanding, every one of them square measure bolstered the brought together methodology that expect the presence of a base station, and exclusively the base station has the power to reinvent indicator hubs, as appeared inside the higher figure in Fig. 1. The brought together methodology isn't dependable on the grounds that, when the base station fizzles or once some identifier hubs lose associations with the base station, it's unrealistic to do reinventing. Besides, there square measure WSNs having no base station at all, and consequently, the brought together methodology isn't appropriate. Additionally, the brought together methodology is wasteful, pitifully adaptable, and vulnerable to some potential assaults on the long correspondence way. On the other hand, as appeared inside the lower figure in Fig. 1,a conveyed approach are frequently used for reinventing in WSNs. It allows numerous affirmed organize clients to simultaneously and legitimately update code pictures on entirely unexpected hubs while not including the base station. Another bit of leeway of conveyed reconstructing is that very surprising endorsed clients might be delegated entirely unexpected benefits of reinventing locator hubs.

As of late, $\mathrm{He}$ et al. have arranged a safe and appropriated reconstructing convention named EDASP [21], 
which is the primary work of its sort. Since a remarkable character based mark plot is utilized in producing open/private key attempt of each endorsed client, EDASP is practical for asset constrained sensor hubs and cell phones as far as correspondence and capacity needs. in addition, EDASP can do all needs of circulated reinventing recorded in [21], while keeping the merits of the notable instruments, for example, Deluge [22] and Seluge [17]. Additionally, EDASP has been authorized in a system of asset constrained gadget hubs to show its high intensity in watch. Notwithstanding, during this paper, we exhibit that a style we tend to exists inside the client. Framework rundown of unified and circulated reconstructing approaches. preprocessing part of EEDASP, and an individual will essentially mimic any affirmed client to hold out reconstructing .To dispense with the known security powerlessness, we proposed straightforward adjustment on EEDASP while not losing any options(such as dispersed reinventing, supporting very surprising client benefits, dynamic cooperation, quantifiability, high strength , and solid security) of the underlying convention

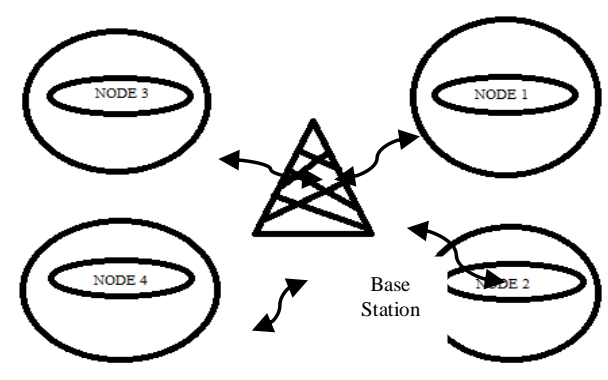

Figure 1 System overview of WSN approaches

In addition, we tend to show that, for security and strength thought, any efficient personality based mark recipe that has endure a couple of long stretches of open examination are frequently straightforwardly utilized in EEDASP. This paper conjointly reports the trial consequences of the improved EEDASP in PC and asset constrained sensor hubs that show its intensity in follow.

\section{BRIEF OVERVIEW OF EEDASP}

The first section of the design is to understand the existing and proposed schemes and how the existing system results have been demonstrated by means of simulation so that the similar strategy can be applied over the proposed scheme. The EDASP consists of three phases: System initialization, User preprocessing, Sensor node verification. In the system initialization section, the network owner creates its public and private keys so assigns the reprogramming privilege and the corresponding non-public key to the approved user(s).

\section{A. System Low-Level Formatting Phase}

The network owner executes the subsequent steps.
1) Let $G$ be a cyclic additive cluster and GT be a cyclic increasing cluster of an equivalent primer order letter of the alphabet. Let $P$ be a generator of G. Let ${ }^{\wedge} \mathrm{e}: \mathrm{G} \times \mathrm{G} \rightarrow \mathrm{GT}$ be a bilinear map.

2) Choose random $s \in Z * q$ because the passe-partout, and reckon public key $\mathrm{PK}$ owner $=\mathrm{s} \bullet \mathrm{P}$.

3) Select 2 secure crypto logical hash functionsH1 and H2, wherever $\mathrm{H} 1: * \rightarrow \mathrm{G}$ andH2: $* \rightarrow \mathrm{Z} * \mathrm{q}$. Then, the general public parameters are loaded in every sensor node before preparation.

4) For a user $\mathrm{Uj}$ with identity $\operatorname{UIDj} \in *$, the network owner sets $\mathrm{Uj}$ 's public key as $\mathrm{PK} \mathrm{j}=\mathrm{H} 1$ (UIDjPrij) $\in \mathrm{G}$, computes the non-public key $\mathrm{SKj}=\mathrm{S} \cdot \mathrm{PKj}$, so sends back to $\mathrm{Uj}$.

\section{B. User preprocessing Phase:}

User Uj takes the subsequent actions.

1) $\mathrm{Uj}$ partitions the code image to $\mathrm{Y}$ fixed-size pages, denoted as page one through page $\mathrm{Y}$. Uj splits page $\mathrm{i}(1 \leq$ $\mathrm{i} \leq \mathrm{Y}$ ) into $\mathrm{N}$ fixed-size packets, denoted as Pkti,1 through Pkti, N . The hash price of every packet in page $\mathrm{Y}$ is appended to the corresponding packet in page $\mathrm{Y}-$ one. For example, the hash price of packet $\mathrm{PktY}, 1 \mathrm{~h}(\mathrm{PktY}, 1)$ is included in packet PktY $-1,1$. Here, PktY, 1 presents the first packet of page Y. Similarly, the hash price of every packet in page $\mathrm{Y}-$ one is enclosed within the corresponding packet in page $\mathrm{Y}-$ a pair .This method continues till $\mathrm{Uj}$ finishes hashing all the packets in page a pair of and as well as their hash values within the corresponding packets in page one. Then, a Merkle hash tree [23] is employed to facilitate the authentication of the hash values of the packets in page one. We talk over with the packets associated with this Merkle hash tree collectively as page zero. The foundation of the Merkle hash tree, the information regarding the code image (e.g., version variety, targeted node identity set, and code image size), and a signature over all of them are enclosed during a signature. Assume that the message $m$ represents the foundation of the Merkle hash tree and also the information regarding the code image. Then, so as to make sure the legitimacy and integrity of the new code image, $\mathrm{Uj}$ takes the subsequent actions to construct the signature message.

2) With the personal key $\mathrm{SKj}, \mathrm{Uj}$ will calculate the signature $\sigma \mathrm{j}$ of the message $\mathrm{m}$, wherever $\sigma \mathrm{j}=\mathrm{H}_{2}(\mathrm{~m}) \cdot \mathrm{SKj}$

3) Uj transmits to the targeted nodes the signature message that is the notification of the new code image. EDASP depends on the underlying Deluge protocol to distribute packets for a given code image. Moreover, we have a tendency to show that, for security and potency thought, any economical identity- based signature algorithmic program that has survived a few years of public scrutiny is directly 
employed in EDASP. This paper additionally reports the experimental results of the improved EDASP in portable computer PCs and resource limited sensor nodes, that show its potency in observe. The remainder of this paper is organized.

\section{Sensor Node Verification}

Upon receiving a signature message, each sensing element node verifies it as follows.

1) The sensing element node initial pays attention to the lawfulness of the programming privilege Prij and also the message $\mathrm{m}$. Only if they're valid, the verification procedure goes to the next step.

2) Given the general public parameters, the sensing element node performs the following verification: ${ }^{\wedge} \mathrm{e}(\sigma \mathrm{j}, \mathrm{P})=$ e (H2(m) • H1(UIDj|Prij), PKowner) . (1) If the equation holds, the signature $\sigma \mathrm{j}$ is valid.

3) If the said verification passes, the sensing element node believes that the message $\mathrm{m}$ and also the privilege Prij square measure from a licensed user with identity UIDj. Hence, the sensor node accepts the basis of the Merkle hash tree constructed for page zero. Thus, the nodes will evidence the hash packets in page zero once they receive such packets, based on the protection of the Merkle hash tree. The hash packets embrace the hash values of the information packets. Therefore, when verification the hash packets, a node can simply verify the information packets in page one supported the unidirectional property of hash functions. Likewise, once the data packets in page I even have been verified, the information packets in page $\mathrm{i}+1$, wherever $\mathrm{i}=$ one, $2, \mathrm{Y}-1$. Given that all verification procedures delineate antecedently pass, the sensing element node accepts the code image. Only the public parameters are loaded on every sensor node before deployment. In the user preprocessing section, if a network user enters the WSN and incorporates a new code image, it will got to construct the reprogramming packets so send them to the sensor nodes. Within the sensing element node verification section, if the packet verification passes, then the nodes settle for the code image.

\section{OPPORTUNITIES TO APPLY WSNS IN NETWORK SIMULATOR}

\section{A. Network Admin}

The network vendor allows register the users and handing over the privilege to set of sensor nodes. The user needs the privilege to access its neighbor sensor nodes. The vendor allows to user can reprogram without admin involved. The network owner creates public and private key has to be created for secure purpose of the sensor nodes.

\section{B. User Preprocessing}

The network vendor set the privilege for the user and calculates the hash worth of every packet within the page is additional to the packet. The user has got to give signature for overall pages to make sure authentication. The message ought to contain the reprogramming privileges then targeted node identity set field indicates the identities of the sensing element nodes that the network user needs to reprogram. Partition the code image and add the signature through the code image.

\section{Node Categorization}

The user verify that whether or not the sensor node must the malicious behavior or not and infected node known as adversaries by using the following technique.

- Nodes infected at time $t$ might infect alternative nodes in the Future

- The finishing fraction of the infected nodes depends on the organization criterion,

- For large H only rare will be infected.

- For small sufficient $\mathrm{H}$ all nodes will be infected.

\section{Check User Privileges}

The sensor node instructions the user privilege to analyses the actual user has the privilege to reprogram that sensor node and primarily pays attention to the validity of the programming privilege and also the message. The individuality of that exact sensor node is present in the privilege list of the user or not. Uncertainty, current in the sense the system public parameters allocated by the network owner is verified when the verification the sensor node trusts that, the code image is since the authenticated user and the sensor node verifies the data packets in the code image.

\section{E. Data Packet Verification}

A Merkle tree is a tree during which each non-leaf node is considered with the hash of the labels of its children nodes. The sensor nodes can authenticate the hash packets in page 0 once the nodes receive such packets, based on the security of the Merkle hash tree. Once the data packets in page $\mathrm{i}$ have been verified a sensor node can easily authenticate the data packets in the page. Only if all verification processes described already pass, the sensor node receives the code image.

\section{F. Energy Based User Selection:}

As Wireless sensor networks become more and more crucial to the normal functioning of people and organizations, availability faults become fewer tolerablelack of availability can make the difference between business as usual and lost productivity, power outages, environmental disasters, and even lost lives; thus high availability of these networks is a critical property, and ought to hold even under malicious conditions. The 
allocation of the Users in EDASP is a significant task as the failure of the User Node might cause the failure of the entire group of nodes under that particular User. Considering the energy of each wireless sensor node the allocation of the users can be done to enhance the overall energy efficiency of the EDASP without altering the multi- authorization of the network.

\section{Method: \\ - User will be selected based on the energy level of the node in the network \\ - The appropriate selection of the user will increase the efficiency of the entire network while the confidentiality remains to the same quality in the network.}

\section{G. Performance Comparison:}

The existing EDASP and the proposed Energy EfficientEDASP with an augmentation on the Energy Efficient User Selection are evaluated for the energy conservation. The intermediate nodes cannot misuse the forwarding information or interpret the data. Simulation of the EDASP and EE-EDASP in network simulator has provided a comparison of throughput, delay and loss in the system

\section{IMPROVEMENT OF SECURITY EDASP}

Obviously, if $\mathrm{H} 2(\mathrm{~m})$ and $\mathrm{Q}$ aren't coprime, Associate in Nursing someone cannot figure the personal key $\mathrm{SKj}$. Therefore, the design weakness of the user preprocessing section doesn't exist, and the ensuing attack is invalid. to attain this goal, the subsequent step is recommended to be more into EDASP. In the system initiation, the order Q of cyclic additive group $\mathrm{G}$ and cyclic increasing cluster GT ought to be set to a large number. Note that Boneh et al. have introduced composite-order linear teams [24], that are wont to successfully solve several difficult issues in cryptography .In the user preprocessing section, once user $\mathrm{Uj}$ computes $\mathrm{m}$, it can check whether or not $\mathrm{H} 2(\mathrm{~m})$ and $\mathrm{Q}$ square measure coprime.

If before a signature on $\mathrm{m}$ is computed, redundant bits square measure appended into m such $\mathrm{H} 2(\mathrm{~m})$ and $\mathrm{Q}$ aren't coprime; otherwise, as described in Section II-B, user Uj directly computes a signature on $\mathrm{m}$. On the opposite hand, the detector node verification section remains a similar. That is, compared to the first EDASP, the recommended modification doesn't incur any overhead on the sensor node aspect. In the style of EDASP, the length of $m$ is twenty nine $\mathrm{B}$. conjointly assume that the hash operate $\mathrm{H} 2$ is enforced victimization SHA-1 with a 20-B output. Taking Q as a 160-b random number, we carry out experiments of coprime checking on laptop computer PCs with totally different machine powers. In every experiment, $q$ is randomly generated for a thousand times. For each $\mathrm{q}, \mathrm{m}$ is indiscriminately generated for a thousand times.
Thus, every experiment has a million measurements. The experimental results show that, without the addition of any redundant bit, the chance that $\mathrm{H} 2(\mathrm{~m})$ and $\mathrm{Q}$ aren't coprime is fifty eight.0212\%. Also, our implementation results regarding the common search time of acceptable redundant data and therefore the failure rate with the addition of 1 or 2 redundant bytes square measure summarized. Here, we tend to take into account a $1.6-\mathrm{GHz}$ processor and therefore the addition of 1 redundant computer memory unit as Associate in nursing example. The failure rate for looking acceptable redundant data is $0.4597 \%$ for this experiment (i.e., the chance that $\mathrm{H} 2$ (m) and Q aren't coprime is one - zero.4597\% $=99.5403 \%$ ), and the search of acceptable redundant knowledge is extremely quick (i.e., the average execution time is sixty eight.12 $\mu \mathrm{s}$ ). Clearly, failure rates only rely on the bit length of the more redundant knowledge however not on processor speed. Furthermore, taking Q as a 160-b random even range, we repeat the aforesaid experiments of coprime checking. The experimental results show that, while not the addition of any redundant bit, the chance that $\mathrm{H} 2(\mathrm{~m})$ and $\mathrm{Q}$ aren't coprime is $59.4491 \%$. Also, with the addition of 1 or 2 redundant bytes, the failure rates for looking acceptable redundant knowledge are all zero for every experiment (i.e., the chance thatH2(m) and Q aren't coprime is 100\%). On the opposite hand, our implementation results regarding the common search time of acceptable redundant knowledge of one or two B square measure summarized. It can be seen that the search of acceptable redundant knowledge is extremely fast. for instance, with the addition of 1 redundant computer memory unit, the average execution times square measure forty. 38 and $36.50 \mu$ s on 1.6- and 1.8-GHz laptop computer PCs, severally. Here, it's recommended to solely use one redundant computer memory unit once Q could be a $160-b$ random even range. With this setting, not solely zero failure rate is achieved however conjointly several benefits within the user preprocessing procedure square measure obtained in terms of computation, memory usage, and transmission and reception powers.

Table I. Running Time for Each Phase of the Improved EDASP (Except the Sensor Node Verification Phase)

\begin{tabular}{cccc} 
CPU Time & Key setup & $\begin{array}{l}\text { User } \\
\text { key generation }\end{array}$ & User signing \\
\hline $1.6 \mathrm{GHz}$ & 5809.5 & 1416.5 & 6518.5 \\
$1.8 \mathrm{GHz}$ & 5074.5 & 1250 & 5707.5 \\
& & & 5111 \\
$2 \mathrm{GHz}$ & 4396.5 & 975.5 & \\
$2.2 \mathrm{GHz}$ & 4233 & 842 & 4641
\end{tabular}


Table II. Implementation of signature messages in the original EDASP and the improved EDASP

\begin{tabular}{llll} 
Value & & The Initial EDASP & $\begin{array}{l}\text { The Improved } \\
\text { EDASP }\end{array}$ \\
\hline \multirow{2}{*}{ Telos B } & ROM & 28,990 & 21,540 \\
& ROM & 600 & 901 \\
& ROM & 25330 & 23,216 \\
Mica Z & ROM & 530 & 986
\end{tabular}

\section{FURTHER IMPROVEMENT OF EDASP}

Designing a secure reprogramming protocol could be a troublesome task, as a result of its square measure such a big amount of details concerned (e.g., the difficult interactions with the environment) that the designer will only strive his/her best to form certain his/her protocol is unfailing. This holds no matter whether or not security proofs square measure supported by heuristic arguments or formal ways in which. In reality, the degree of confidence concomitant a security mechanism will increase with time provided that the underlying algorithms will survive a few years of public scrutiny.

EDASP is predicated on a completely unique and fresh designed identity based signature rule. the easy modification given will fix the known security drawback of this signature rule, however it's still unsure whether or not there's any other security weakness during this changed identitybased signature rule. to deal with this issue, it's prompt that, instead of this novel identity-based signature rule, some efficient identity-based signature algorithms that have survived many years of public scrutiny will be directly used in EDASP. For instance, we are able to opt for the incontrovertibly secure identity-based signature planned by Barreto et al. Aside from providing higher security, the tactic by Barreto et al. also improves the potency of EDASP attributable to the subsequent 2 reasons. First, its signature verification operation solely wants one pairing computation and, hence, is among the foremost economical ones. Second, the length of its signature is reduced attributable to bilinear pairing.

A) System low-level formatting Phase: The network owner executes the following steps.

1) Key setup: Generate the general public parameters params $=$, and load them in every device node before readying, where $(\mathrm{G} 1, \mathrm{G} 2, \mathrm{G} 3)$ represents linear teams of large prime order $\mathrm{p}$ with generators $\mathrm{g} 2 \in \mathrm{G} 2, \mathrm{~g} 1=\psi(\mathrm{g} 2) \in$ $\mathrm{G} 1$, and $\mathrm{g}={ }^{\mathrm{e}}(\mathrm{g} 1, \mathrm{~g} 2)$. The network owner picks a random variety $\mathrm{s} \in \mathrm{Zp}$ because the master and computes public key Qpub = $\mathrm{s} \cdot \mathrm{g} 2 \in \mathrm{G} 2$. H3 and H4 are cryptographic hash functions, wherever $\mathrm{H} 3: * \rightarrow \mathrm{Zp}$ and $\mathrm{H} 4$ : $* \times \mathrm{G} 3 \rightarrow \mathrm{Z} * \mathrm{p}$.

2) User public/private key generation: For a user $\mathrm{Uj}$ with identity $\mathrm{UIDj} \in *$, the network owner sets $\mathrm{Uj}$ 's public key as $\mathrm{Pj}=\mathrm{H} 3$ (UIDj Prij) $\in \mathrm{Z} * \mathrm{p}$, computes the non-public key $\mathrm{Sj}=(1 /(\mathrm{Pj}+\mathrm{s})) \cdot \mathrm{g} 1=(1 /(\mathrm{H} 3(\mathrm{UIDj}$ Prij $)+\mathrm{s})) \cdot \mathrm{g} 1$, so sends back to Uj through a secure channel. Here, Prij denotes the amount of user privilege (e.g., the sensor node set among a selected region that user $\mathrm{Uj}$ is allowed to reprogram) and subscription amount.

\section{B) User Preprocessing Phase:}

User $\mathrm{Uj}$ takes the subsequent actions.

3) This step is that the same as step 1) of the user preprocessing phase of the first EDASP.

4) With the non-public key $\mathrm{Sj}, \mathrm{Uj}$ will cipher the signature $\sigma j$ of the message $m$ as represented within the following. Pick a random variety $x \in Z * p$, and cipher $r=$ gx. Set $h=$ $\mathrm{H} 4(\mathrm{~m}, \mathrm{r}) \in \mathrm{Z} * \mathrm{p}$, and cipher $\mathrm{W}=(\mathrm{x}+\mathrm{h}) \cdot \mathrm{Sj}$. The signature $\sigma \mathrm{j}$ is that the combine $(\mathrm{h}, \mathrm{W}) \in \mathrm{Z} * \mathrm{p} \times \mathrm{G} 1$.

5) This step is that the same as step 3) of the user preprocessing phase of the first EDASP.

C) Device Node Verification Phase: Upon receiving a signature message, every device node verifies it as follows.

1) This step is that the same as step 1) of the device node verification part of the first EDASP.

2) Given the general public parameters, the device node computes $\mathrm{h} *=\mathrm{H} 4 \mathrm{~m}$, e (W, H3 (UIDjPrij) $\bullet \mathrm{g} 2+$ Qpub) $\mathrm{g}-\mathrm{h}$ and then sees whether or not $\mathrm{h} *$ is adequate to $\mathrm{h}$ or not, where $\mathrm{h}$ is from $\sigma \mathrm{j}$. If the result's positive, the signature $\sigma \mathrm{j}$ is valid; otherwise, the node merely drops the signature.

3) This step is that the same as step 3) of the device node verification part of the first EDASP.

\section{CONCLUSION}

Previously quantity of secure reprogramming protocols are predictable. However none of those approaches support distributed operation .In my project secure distributed reprogramming protocol named EDASP with node classification rule has been proposed. Additionally to analyzing the protection of EDASP, I according the analysis results of EDASP by exploitation the Network machine Ns2 with network of resource- limited sensing element nodes that shows that EDASP is possible in apply. To the simplest of our data, until now, our protocol the sole one that enables approved users to reprogram sensor nodes during a distributed manner and additionally classify the sensor nodes before causing the code image to the sensing element 
node. So our projected protocol provides a lot of applications, security whereas reprogramming the sensor nodes

\section{REFERENCES}

[1] Daojing He, Student Member, IEEE, Chun Chen, Member, IEEE, Sammy Chan, Member, IEEE, Jiajun Bu, Member, IEEE, and Laurence T. Yang, Member, IEEE\| Security Analysis and Improvement of a Secure and Distributed Reprogramming Protocol for Wireless Sensor Networks\| IEEE Transactions On Industrial Electronics, Vol. 60, No. 11, November 2013.

[2] V. C. Gungor and G. P. Hancke, -Industrial wireless sensor networks: Challenges, design principles, and technical approaches, IEEE Trans. Ind. Electron., vol.56, no. 10, pp. 4258-4265, Oct. 2009.

[3] V. C. Gungor, B. Lu, and G. P. Hancke, Opportunities and challenges of wireless sensor networks in smart grid,„IEEE Trans. Ind. Electron.,vol. 57, no. 10, pp. 3557-3564, Oct. 2010

[4] J. Chen, X. Cao, P. Cheng, Y. Xiao, and Y. Sun, Distributed collaborative control for industrial automation with wireless sensor and actuator networks, IEEE Trans. Ind. Electron., vol. 57, no. 12, pp. 4219-4230, Dec. 2010.

[5] X. Cao, J. Chen, Y. Xiao, and Y. Sun, -Building- environment control with wireless sensor and actuator networks: Centralized versus distributed,॥ IEEE Trans. Ind. Electron., vol. 57, no. 11, pp. 3596-3604, Nov. 2010.

[6] J. Carmo, P. Mendes, C. Couto, and J. Correia, -A 2.4-GHz CMOS shortrange wireless-sensor-network interface for automotive applications,\| IEEE Trans. Ind. Electron., vol. 57, no. 5, pp. 1764-1771, May 2010.

[7] V. Naik, A. Arora, P. Sinha, and H. Zhang, - Sprinkler: A reliable and energy efficient data dissemination service for extreme scale wireless networks of embedded devices,\| IEEE Trans. Mobile Comput., vol. 6,no. 7, pp.762-776, Jul. 2007.

[8] L.Mottola and G. Picco, - Programming wireless sensor networks: Fundamental concepts and state of the art, ॥ ACM Comput. Surv., vol. 43, no. 3,pp. 1-51, Apr. 2011.

[9] H. Song, V. Shin, and M. Jeon, - Mobile node localization using fusionprediction-based interacting multiple model in cricket sensor network,\| a. IEEE Trans. Ind. Electron. vol. 59, no. 11 , pp. 4349-4359, Nov. 2010.

[10] R. C. Luo and O. Chen, - Mobile sensor node deployment and asynchronous power management for wireless sensor networks,\| IEEE Trans. Ind. Electron. vol. 59, no. 5, pp. 23772385, May 2012.

[11] H. Tan, J. Zic, S. Jha, and D. Ostry, -Secure multihop network programming with multiple one-way key chains,\| IEEE Trans. Mobile Comput.,vol. 10, no. 1, pp. 16-31, Jan. 2011.

[12] P. K. Dutta, J. W. Hui, D. C. Chu, and D. E. Culler, - Securing the deluge network programming system, $\|$ in Proc. IPSN, 2006, pp. 326-333.

[13] C. Lim, - Secure code dissemination and remote image management using short-lived signatures in WSNs,\| IEEE Commun. Lett., vol. 15, no. 4, pp. 362-364, Apr. 2011.

[14] I. Doh, J. Lim, and K. Chae, —Code updates based on minimal backbone and group key management for secure sensor networks, $\|$ Math. Comput.Model., 2012, to be published.

[15] Y. Law, Y. Zhang, J. Jin, M. Palaniswami, and P. Havinga, - Secure rateless deluge: Pollution-resistant reprogramming and data dissemination for wireless sensor networks,\| EURASIP J. Wireless Commun. Netw.,vol. 2011, no. 1, pp. 1-21, Jan. 2011.
[16] C. Parra and J. Garcia-Macias, -A protocol for secure and energy-aware reprogramming in WSN,\| in Proc. IWCMC, 2009, pp. 292-297.

[17] N. Bui, O. Ugus, M. Dissegna, M. Rossi, and M. Zorzi, -An integrated system for secure code distribution in wireless sensor networks, $\|$ in Proc.PERCOM, 2010, pp. 575-581.

[18] S. Hyun, P. Ning, A. Liu, and W. Du, -Seluge: Secure and DoS-resistant code dissemination in wireless sensor networks, $\|$ in Proc. IPSN, 2008, pp. 445-456.

IEEE Transactions on Industrial Electronics, Vol. 60, No. 11, November 2013

[19] D. He, S. Chan, C. Chen, and J. Bu, - Secure and efficient dynamic program update in wireless sensor networks, $\|$ Secur. Commun. Netw., vol. 5,no. 7, pp. 823-830, Jul. 2012.

[20]Geoss, 2011. [Online]. Available: http://www.epa.gov/geoss/. 